Pacific Journal of Mathematics

INTEGRAL REPRESENTATION OF TCHEBYCHEFF SYSTEMS 


\title{
INTEGRAL REPRESENTATION OF TCHEBYCHEFF SYSTEMS
}

\author{
R. A. ZALIK
}

A representation of Tchebycheff systems in terms of iterated Riemann-Stieltjes integrals, is given.

1. Introduction. A system of real-valued functions $\left\{u_{0}, u_{1}, \cdots, u_{n}\right\}$ defined on a totally ordered set is called a Tchebycheff system or $T$-system (Weak Tchebycheff system or $W T$-system), provided that for every choice of points $t_{0}<t_{1}<\cdots<t_{n}$ of the set,

$$
D\left(u_{0}, u_{1}, \cdots, u_{n} / t_{0}, t_{1}, \cdots, t_{n}\right)=\operatorname{det}\left(u_{i}\left(t_{j}\right) ; i, j=0,1, \cdots, n\right)
$$

is strictly positive (nonnegative). A function $u$ is said to be convex with respect to the system $\left\{u_{0}, u_{1}, \cdots, u_{n}\right\}$, if $\left\{u_{0}, u_{1}, \cdots, u_{n}, u\right\}$ is a $W T$ system. The set of functions convex with respect to $\left\{u_{0}, u_{1}, \cdots, u_{n}\right\}$ is evidently a cone. This cone is referred to as "Generalized Convexity Cone". If $\left\{u_{0}, u_{1}, \cdots, u_{i}\right\}$ is a $T$-system for $i=0,1, \cdots, n$, then $\left\{u_{0}, u_{1}, \cdots, u_{n}\right\}$ is called a Complete Tchebycheff system or CTsystem. Note that no assumptions of continuity have been made in this paragraph.

In 1965 there appeared a paper by M. A. Rutman in which the following proposition is stated (cf. [4, Thm. 3]):

THEOREM. Suppose the system of right-continuous functions $\left\{1, u_{1}, u_{2}, \cdots, u_{n}\right\}$ is a $C T$-system on the open interval $(a, b)$. Then.there is a system $\left\{1, y_{1}, y_{2}, \cdots, y_{n}\right\}$ admitting of the following two representations on $(a, b)$ :

$$
y_{i}=u_{i}+\sum_{j=0}^{i-1} a_{i, j} u_{j} ; \quad i=1,2, \cdots, n,
$$

and

$$
\begin{aligned}
& y_{1}(t)=\int_{c}^{t} d p_{1}(s) \\
& y_{2}(t)=\int_{c}^{t} \int_{c}^{s_{1}} d p_{2}\left(s_{2}\right) d p_{1}\left(s_{1}\right) \\
& y_{n}(t)=\int_{c}^{t} \int_{c}^{s_{1}} \cdots \int_{c}^{s_{n-1}} d p_{n}\left(s_{n}\right) d p_{n-1}\left(s_{n-1}\right) \cdots d p_{1}\left(s_{1}\right),
\end{aligned}
$$

where $c \in(a, b)$ is arbitrary, and the functions $p_{1}$ are strictly increasing and right-continuous on $(a, b)$. 
Rutman's theorem has apparently nowhere been proved in detail; in fact, as will be shown in the next section, it is not correct with this degree of generality. The purpose of this paper is to investigate the existence of representations of the type (3), in the most general context possible.

If the functions $u_{\imath}$ are $n$ times continuously differentiable on an interval $I$, we can extend the definition of $D\left(u_{0}, \cdots, u_{n} / t_{0}, \cdots, t_{n}\right)$ as given by (1), so as to allow for equalities amongst the $t_{t}:$ if $t_{0} \leqq t_{1} \leqq \cdots \leqq t_{n}$ is any set of points of $I$, then $D^{*}\left(u_{0}, \cdots, u_{n} / t_{0}, \cdots, t_{n}\right)$ is defined to be the determinant in the right hand side of (1), where for each set of equal $t_{i}$ 's, the corresponding columns are replaced by the successive derivatives evaluated at the point. With this definition, the system $\left\{u_{0}, \cdots, u_{n}\right\}$ will be called an Extended Tchebycheff system (ET-system), provided that $D^{*}\left(u_{0}, \cdots, u_{n} / t_{0}, \cdots, t_{n}\right)>0$ for every set $t_{0} \leqq t_{1} \leqq \cdots \leqq t_{n}$ of points of I. If, moreover, the systems $\left\{u_{0}, \cdots, u_{i}\right\} ; i=0,1, \cdots, n$ are $E T$-systems, the system $\left\{u_{0}, \cdots, u_{n}\right\}$ is called an Extended Complete Tchebycheff system (ECT-system). The validity of Rutman's theorem for ECTsystems has essentially been proved in [3, Ch. XI, Theorem 1.2], a fact which will be used further along in our discussion.

We now turn to the statement of our results. Following R. Zielke $[8,9]$, we shall say that a set $A$ has property (D), provided it is totally ordered, it contains no smallest nor greatest element, and for every two distinct elements of $A$, there is a third element of $A$ in between. The main feature of this paper is the following:

THEOREM 1. Let $\left\{u_{0}, \cdots, u_{n}\right\}$ be a CT-system on a nondenumerable set $A$ having property (D), and let $c \in A$. Then there is a system of functions $\left\{y_{0}, \cdots, y_{n}\right\}$ having the following properties:

(a) The functions $y_{1}, \cdots, y_{n}$ have a representation of the form (2) and $y_{0}=u_{0}$ on $A$.

(b) There is a subset $B$ of $A$, having an at most denumerable complement in $A$, a real valued strictly increasing function $h$, defined on $A$, and a set $\left\{p_{1}, \cdots, p_{n}\right\}$ of real valued strictly increasing functions, defined on the open interval whose end points are the infimum and the supremum of $h(A)$, such that $p_{i}[h(c)]=0, i=1, \cdots, n$ and, for every point $t$ of $B$,

$$
\begin{aligned}
& y_{1}(t)=y_{0}(t) \int_{h(c)}^{h(t)} d p_{1}(s) \\
& y_{2}(t)=y_{0}(t) \int_{h(c)}^{h(t)} \int_{h(c)}^{s_{1}} d p_{2}\left(s_{2}\right) d p_{1}\left(s_{1}\right) \\
& y_{n}(t)=y_{0}(t) \int_{h(c)}^{h(t)} \int_{h(c)}^{s_{1}} \cdots \int_{h(c)}^{s_{n-1}} d p_{n}\left(s_{n}\right) d p_{n-1}\left(s_{n-1}\right) \cdots d p_{1}\left(s_{1}\right) .
\end{aligned}
$$


Moreover, if the function $y$ is convex with respect to the system $\left\{u_{0}, \cdots, u_{n}\right\}$, it admits of the representation

$$
y(t)=u(t)+y_{0}(t) \int_{h(c)}^{h(t)} \int_{h(c)}^{s_{1}} \cdots \int_{h(c)}^{s_{n}} d p_{n+1}\left(s_{n+1}\right) \cdots d p_{1}\left(s_{1}\right)
$$

on $B$, where $p_{n+1}$ is an increasing function and $u$ is in the linear span of the system $\left\{u_{0}, \cdots, u_{n}\right\}$.

(c) For $i=1, \cdots, n$, the functions $y_{i}$ are uniquely determined, and the functions $p_{i}$ are uniquely determined (a.e.), by the functions $h, u_{0}, \cdots, u_{i}$.

(d) If $A$ is a dense subset of an open interval (in particular if $A$ is an open interval), $h$ can be taken to be the identity function: $h(t)=t$.

(e) Let $v^{+}(t)$ and $v^{-}(t)$ denote the right and left one-sided limits of the function $v$ at the point $t$, and let $v_{t}=\left(u_{i} \circ h^{-1}\right) /\left(u_{0} \circ h^{-1}\right)$. If $t$ is an element of the set $B$ there is a real number $p$, contained in the interval $[0,1]$, such that if $s=h(t)$, then

$$
z_{\imath}(s)=p z_{i}^{+}(s)+(1-p) z_{i}^{-}(s), \quad i=1, \cdots, n+1 .
$$

Conversely, if for some point $t$ of $A$ the functions $z_{1}$ admit of $a$ representation of the form (6), with $s=h(t)$, then $t$ is contained in $B$, provided that $p$ be contained in the open interval $(0,1)$. If $p=0$ or $p=1$, $t$ may, or may not, belong to $B$. (See the first counterexample in the next section.)

Remarks. (a) Note that if $\left\{u_{0}, \cdots, u_{n}\right\}$ is a $T$-system defined on a set having property (D), its linear span contains a basis that constitutes a $C T$-system, (see [8]).

(b) For the case of an ECT-system, the representation (5) is implicit in [3, Ch. XI, section 11] (see also [6]).

In the following theorem, we have gathered several propositions of independent interest, some of which will be employed in the sequel.

Theorem 2. Let $\left\{1, y_{1}, \cdots, y_{n}\right\}$ be a CT-system on a dense subset $A$ of an open interval $I$, and assume that $y_{n+1} \in C\left(1, y_{1}, \cdots, y_{n}\right)$ thereon. Then:

(a) For $i=1, \cdots, n+1$, and every point $t$ of $I$, the one-sided limits $y_{i}^{+}(t)$ and $y_{i}^{-}(t)$ exist and are finite, and the functions $y_{i}^{+}$and $y_{i}^{-}$thus defined are of bounded variation in every closed subinterval of $I$.

(b) For every function $\alpha: I \rightarrow[0,1]$, if $z_{i}=\alpha y_{i}^{+}+(1-\alpha) y_{i}^{-}$, then $\left\{1, z_{1}, \cdots, z_{n}\right\}$ is a CT-system on $I$, and $z_{n+1} \in C\left(1, y_{1}, \cdots, y_{n}\right)$ thereon, under the additional assumption that $A$ is nondenumerable.

(c) If $y_{1}$ is right (left) continuous at a given point of $A$, all the functions $y_{i}, i=1, \cdots, n+1$ are right (left) continuous at this point.

(d) Let $n>1$, and let $s$ be a point of I. If equation (6) is satisfied by 
the functions $y_{1}, y_{2}$, and $y_{3}$, where $p$ is a point of the interval $[0,1]$, then it is satisfied (for the same number $p$ ) by all the functions $y_{t}, i=1, \cdots, n+1$.

Remark. Note the connection between Theorem $2 \mathrm{~d}$. and Theorem 1e.

The proof of Theorem 1 will be divided into two parts, which we shall now outline. In the first part, we shall consider bounded functions $u_{t}$ defined on an open bounded interval $I$, such that $\left\{1, u_{1}, \cdots, u_{n}\right\}$ is a $C T$-system, $u_{n+1} \in C\left(1, u_{1}, \cdots, u_{n}\right)$ and, for $i=1, \cdots, n+1, u_{i}=(1 / 2)$. $\left(u_{t}^{+}+u_{i}^{-}\right)$. Convolving each function $u_{t}$ with the Gauss kernel $G_{k}(s)$ we obtain (for each $k$ ), an ECT-system $\left\{u_{0}(k, \cdot), \cdots, u_{n}(k, \cdot)\right\}$, such that $u_{n+1} \in C\left(u_{0}(k, \cdot), \cdots, u_{n}(k, \cdot)\right)$. As we have already remarked, Rutman's Theorem is valid for ECT-systems. Since $u_{t}=(1 / 2)\left(u_{t}^{+}+u_{i}^{-}\right)$, it can be shown that $\lim _{n \rightarrow \infty} u_{i}(k, \cdot)=u_{t}$ on $I$. Thus, the proof of this case will follow (after a number of steps), by letting $k$ tend to infinity. The general case will be considered in the second part of the proof. By a suitable normalization, the original system will be transformed into a $C T$-system of bounded functions defined on a dense subset $D$ of an open interval $I$. Redefining these functions so that they will equal the average of their lateral limits everywhere on $D$, and applying Theorem 2 to extend them to the whole of $I$, we shall reduce the problem to the one considered in the first part of the proof.

Using Theorems 1 and 2, we shall easily prove the following proposition, which generalizes a result of Bartelt [1, Theorem 1]:

THEOREM 3. Let $\left\{u_{0}, \cdots, u_{n}\right\}$ be a T-system on a dense subset $A$ of an interval $(a, b)$. Then:

(a) The system $\left\{u_{0}, \cdots, u_{n}\right\}$ can be extended as a $T$-system to the whole of $(a, b)$.

(b) If $\left\{u_{0}^{*}, \cdots, u_{n}^{*}\right\}$ is a system of continuous functions on $(a, b)$, such that $u_{1}^{*}=u_{\imath}$ on $A, i=0, \cdots, n$, the following propositions are equivalent:

(i) The system $\left\{u_{0}^{*}, \cdots, u_{n}^{*}\right\}$ is a T-system on $(a, b)$.

(ii) The linear span of $\left\{u_{0}^{*}, \cdots, u_{n}^{*}\right\}$ contains a function that does not vanish on $(a, b)$.

(iii) The functions $u_{1}^{*}$ cannot all vanish at any one point of $(a, b)$.

Theorem 1 admits of a converse. Indeed, we shall readily prove the following:

LEMMA. If the functions $y_{1}$ admit of a representation of the form (4) on a totally ordered set $A$, where the functions $h, p_{1}, \cdots, p_{n}$ are strictly increasing, and $y_{0}$ is strictly positive, then $\left\{y_{0}, \cdots, y_{n}\right\}$ is a CT-system on A. 


\section{Counterexamples to Rutman's theorem. Let}

$$
p_{1}(t)=\left\{\begin{array}{ll}
t-1, & \text { if } \quad-1<t<0 \\
0, & \text { if } t=0 \\
t+1, & \text { if } 0<t<1
\end{array}, \quad p_{2}(t)=\left\{\begin{array}{lll}
t-1, & \text { if } \quad-1<t<0 \\
t+1, & \text { if } & 0 \leqq t<1
\end{array}\right. \text {, }\right.
$$

and define

$$
y_{1}(t)=\int_{0}^{t} d p_{1}(s)
$$

and

$$
y_{2}(t)=\int_{0}^{t} \int_{0}^{s_{1}} d p_{2}\left(s_{2}\right) d p_{1}\left(s_{1}\right)=\int_{0}^{t}\left[p_{2}(s)-1\right] d p_{1}(s)
$$

Thus,

$$
y_{1}(t)=p_{1}(t)
$$

and

$$
y_{2}(t)=\left\{\begin{array}{lll}
(1 / 2) t^{2}-2 t & \text { on } & (-1,0) \\
(1 / 2) t^{2} & \text { on } & {[0,1)}
\end{array}\right.
$$

We know from our Lemma, that $\left\{1, y_{1}, y_{2}\right\}$ is a $C T$-system on $(-1,1)$. We thus see from Theorem 2 b. or by direct computation, that if

$$
u_{1}(t)=\left\{\begin{array}{lll}
t-1 & \text { on } & (-1,0) \\
t+1 & \text { on } & {[0,1)}
\end{array}\right.
$$

and $u_{2}=y_{2}$, then also $\left\{1, u_{1}, u_{2}\right\}$ is a $C T$-system on $(-1,1)$. Assume now, that there are two functions $v_{1}=u_{1}+a, v_{2}=u_{2}+b_{1} u_{1}+b_{2}$, and two strictly increasing functions $q_{1}, q_{2}$, such that $v_{1}(t)=\int_{0}^{t} d q_{1}(s)$, and $v_{2}(t)=$ $\int_{0}^{t} \int_{0}^{s_{1}} d q_{2}\left(s_{2}\right) d q_{1}\left(s_{1}\right)$. Without any loss of generality, we can assume that $q_{1}(0)=q_{2}(0)=0$; thus $v_{1}=q_{1}=u_{1}+a$. If $t$ is any point of the interval $(-1,1)$ other than 0 , it is clear from their definition that $u_{1}=y_{1}$. Since, as remarked above, $y_{1}=p_{1}$, we conclude that $q_{1}=p_{1}+a$, identically on $(-1,1)$, excepting perhaps at zero. Since the function $q_{2}$ vanishes at 0 , it is readily seen that $v_{2}=\int_{0}^{t} q_{2}(s) d s$. On the other hand, from the integral representation of the functions $y_{t}$, and bearing in mind that $u_{i}=y_{i}$ if $t \neq 0$, we see that, for every point of $(-1,1)$, with the possible exception of 0 , $v_{2}=y_{2}+b_{1} y_{1}+b_{2}=\int_{0}^{t}\left[p_{2}(s)+b_{1}-1\right] d p_{1}(s)+b_{2}$. 
Let $r$ be the saltus function of $p_{1}$, i.e.

$$
r(t)=\left\{\begin{array}{lll}
0 & \text { on } & (-1,0) \\
1 & \text { if } & t=0 \\
2 & \text { on } & (0,1)
\end{array}\right.
$$

Then $p_{1}(t)=(t-1)+r(t)$. Thus, if $t>0$,

$$
v_{2}(t)=\int_{0}^{t}\left[p_{2}(s)+b_{1}-1\right] d s+2\left[p_{2}(0)+b_{1}-1\right]+b_{2} .
$$

From the integral representation of $v_{2}$ in terms of $q_{2}$, we see that $v_{2}$ vanishes at 0 . Passing to the limit in the preceding formula, and bearing in mind that $p_{2}(0)=1$, we conclude that $b_{2}+2 b_{1}=0$. Since $u_{1}(0)=1$, and $u_{2}(0)=0$, from the representation of $v_{2}$ in terms of $u_{1}$ and $u_{2}$ we conclude, on the other hand, that $b_{1}+b_{2}=0$. It is therefore clear that $b_{1}=b_{2}=0$. Thus, if $t>0$,

$$
v_{2}(t)=\int_{0}^{t}\left[p_{2}(s)-1\right] d s=\int_{0}^{t} q_{2}(s) d s
$$

and we conclude that for positive values of $t, q_{2}=p_{1}-1$. Passing to the limit, we readily see that $q_{2}$ is right-continuous at 0 . But, being that $q_{1}=u_{1}+a$, also $q_{1}$ is right continuous at 0 , and therefore the integral $v_{2}(t)=\int_{0}^{t} q_{2}(s) d q_{1}(s)$ cannot exist for negative values of $t$.

We can also prove that, even if the desired representation exists, the functions $p_{2}$ in (3) may not be right-continuous. In order to see this, we can consider, for instance, the following example: Let $q_{1}$ and $q_{2}$ be strictly increasing functions defined in the interval $(-1,1)$. Let $q_{1}$ be rightcontinuous and $q_{2}$ left-continuous, and assume that they are bounded, and have the same set of points of discontinuity; assume moreover that $q_{1}(0)=q_{2}(0)=0, \quad$ and define $\quad u_{1}(t)=\int_{0}^{t} d q_{1}(s), \quad$ and $\quad u_{2}(t)=$ $\int_{0}^{t} \int_{0}^{s_{1}} d q_{2}\left(s_{2}\right) d q_{1}\left(s_{1}\right)$. Clearly $u_{1}=q_{1}$, and $u_{2}(t)=\int_{0}^{t} q_{2}(s) d q_{1}(s)$. Thus also $u_{2}$ is right-continuous on $(-1,1)$.

Assume now there are two functions $y_{1}, y_{2}, y_{1}=u_{1}+a, y_{2}=$ $u_{2}+b_{1} u_{1}+b_{2}$, and two strictly increasing, right-continuous functions $p_{1}$, $p_{2}$, such that, for every $t$ in $(-1,1)$,

$$
y_{1}(t)=\int_{0}^{t} d p_{1}(s)
$$


and

$$
y_{2}(t)=\int_{0}^{t} \int_{0}^{s_{1}} d p_{2}\left(s_{2}\right) d p_{1}\left(s_{1}\right)
$$

Since $u_{1}(0)=y_{1}(0)=0$, we conclude that $a=0$. Thus $d p_{1}=d q_{1}$. Since also $u_{2}(0)=y_{2}(0)=0$, it is clear that $b_{2}=0$, whence combining the two representations of $y_{2}$, we see that

$$
y_{2}(t)=\int_{0}^{t}\left[p_{2}(s)-p_{2}(0)\right] d q_{1}(s)=\int_{0}^{t}\left[q_{2}(s)+b_{1}\right] d q_{1}(s) .
$$

Let $k=b_{1}+p_{2}(0)$. It is clear from the preceding identity, that $q_{2}+k=p_{2}$, on the set of points at which $q_{1}$ is continuous. Since $q_{1}$ and $q_{2}$ have the same set of points of discontinuity, we therefore conclude that also $p_{2}$ and $q_{1}$ have the same points of discontinuity. But $d p_{1}=$ $d q_{1}$. Thus $p_{1}$ and $p_{2}$ have the same points of discontinuity. But this is clearly impossible because, being that both $p_{1}$ and $p_{2}$ are rightcontinuous, it will suffice that the interval $(0, t)$ contain one point of discontinuity of $p_{1}$, for the integral

$$
\int_{0}^{t}\left[p_{2}(s)-p_{2}(0)\right] d p_{1}(s)
$$

not to exist.

Rutman's mistake is due to his belief that, if $\left\{y_{0}, \cdots, y_{n}\right\}$ is a $C T$-system of right-continuous functions on an interval $(a, b)$ with $y_{0}=1$, the functions $z_{t}(t)=\lim _{h \rightarrow 0^{+}}\left[y_{t}(t+h)-y_{t}(t)\right] /\left[y_{1}(t+h)-y_{1}(t)\right]$ not only exist for every point $t$ of $(a, b)$, (which is true), but that in addition, they are right-continuous thereon (cf. op. cit. Thm. 2). This can be disproved by considering the following counterexample:

Let

$$
p_{1}(t)=\left\{\begin{array}{lll}
t-1 & \text { on }(-1,0) \\
t & \text { on }[0,1]
\end{array}, \quad p_{2}(t)= \begin{cases}t-1 & \text { on }(-1,0] \\
t+1 & \text { on }(0,1)\end{cases}\right.
$$

and define: $y_{0}=1, y_{1}=p_{1}, y_{2}=\int_{0}^{t} p_{2}(s) d p_{1}(s)$.

Referring to our Lemma, we see that $\left\{y_{0}, y_{1}, y_{2}\right\}$ is a $C T$-system of right-continuous functions on $(a, b)$. Moreover, if $t \neq 0, z_{2}(t)=$ $p_{2}(t)$. However, it is easy to verify that $z_{2}(0)=0$, while $\lim _{t \rightarrow 0^{+}} z_{2}(t)=$ $\lim _{t \rightarrow 0^{+}} p_{2}(t)=1$. 


\section{Proofs.}

Proof of Lemma. Taking into consideration [3, p. 10, Example 2b.], it will suffice to consider the case $h(t)=t$. From the linearity of the integral we conclude (as in the proof of [3, Ch. XI, Lemma 2.1]), that if $\left\{1, y_{1}, y_{2}, \cdots, y_{l}\right\}$ is a $T$-system and $p$ is a strictly increasing function, then $\left\{1, \int_{c}^{t} 1 . d p, \int_{c}^{t} y_{1} d p, \cdots, \int_{c}^{t} y_{i} d p\right\}$ is a $T$-system, whence the conclusion follows by an obvious inductive procedure.

Proof of Theorem 2. (a) Let $I=(a, b)$. The assertions are obvious for $n=0$. Assume they are true if $n=k$, and let $n=k+1$. It will suffice to carry out the proof for every interval of the form $(c, b)$, $a<c<b$, with $c \in A$. Let $g_{i}=\left[y_{i}-y_{i}(c)\right] /\left[y_{1}-y_{1}(c)\right]$, and $c<t_{1}<$ $\cdots<t_{n+1}<b$. Developing by the first column, we readily see that

$$
D\left(1, y_{1}, \cdots, y_{\imath} / c, t_{1}, \cdots, t_{i}\right)=\left(\prod_{j=1}^{n}\left[y_{1}\left(t_{j}\right)-y_{1}(c)\right]\right) D\left(1, g_{2}, \cdots, g_{\imath} / t_{1}, \cdots, t_{t}\right)
$$

Since $y_{1}$ is strictly increasing, the factors $y_{1}\left(t_{j}\right)-y_{1}(c)$ are strictly positive. We thus conclude that $\left\{1, g_{2}, \cdots, g_{n}\right\}$ is a $C T$-system on $A \cap(c, d)$, and $g_{n+1} \in C\left(1, g_{2}, \cdots, g_{n}\right)$ thereon, whence the assertions readily follow from the inductive hypothesis.

(b) Let $B$ denote the set of points of $A$ at which all the functions $y_{1}$ are continuous. From (a) we know that the set difference $A \sim B$ is at most denumerable; therefore $B$ is dense in $I$. Clearly $y_{1}=z_{1}$ on $B$; thus $\left\{1, z_{1}, \cdots, z_{n}\right\}$ is a $C T$-system on $B$, and $z_{n+1} \in C\left(1, \cdots, z_{n}\right)$ thereon. We shall first prove that, for $i=1,2, \cdots, n+1,\left\{1, z_{1}, \cdots, z_{l}\right\}$ is a $W T$-system on $I$; from this will follow, in particular, that $z_{n+1} \in C\left(1, z_{1}, \cdots, z_{n}\right)$ on $I$. The assertion is obvious if the function $\alpha$ can only assume the values 0 and 1 ; indeed, this simply means that for every point $t$, either $z_{l}(t)=y_{l}^{+}(t), i=1, \cdots, n$, or $z_{l}(t)=y_{l}^{-}(t), i=1, \cdots, n$ whence, since $B$ is dense in $I$, the proof of our claim follows by an obvious limiting process. In the general case, the assertion follows from the preceding discussion, the linearity of the determinant, and the fact that the functions $\alpha$ and $1-\alpha$ are nonnegative. For example, let $a<t_{0}<t_{1}<b$, $\alpha\left(t_{0}\right)=p, \alpha\left(t_{1}\right)=q$. Then, setting $z_{0}=1$, we have: $D\left(1, z_{1} / t_{0}, t_{1}\right)=$ $\operatorname{det}\left\|z_{\imath}\left(t_{0}\right), z_{i}\left(t_{1}\right)\right\|=\operatorname{det}\left\|p z_{i}^{+}\left(t_{0}\right)+(1-p) z_{\imath}^{-}\left(t_{0}\right), \quad q z_{\imath}^{+}\left(t_{1}\right)+(1-q) z_{\imath}^{-}\left(t_{1}\right)\right\|=$ $p q \operatorname{det}\left\|z_{i}\left(t_{0}\right), z_{i}^{+}\left(t_{1}\right)\right\|+p(1-q) \operatorname{det}\left\|z_{i}^{+}\left(t_{0}\right), z_{i}^{-}\left(t_{1}\right)\right\|+$ $(1-p) q \operatorname{det}\left\|z_{i}^{-}\left(t_{0}\right), z_{i}^{+}\left(t_{1}\right)\right\|+(1-p)(1-q) \operatorname{det}\left\|z_{i}^{-}\left(t_{0}\right), z_{i}^{-}\left(t_{1}\right)\right\| \geqq 0$.

To prove that $\left\{1, z_{1}, \cdots, z_{n}\right\}$ is a $C T$-system we proceed by induction. The assertion is obvious if $n=0$. Assume it to be true if $n=k$, and let $n=k+1$. By inductive hypothesis, $\left\{1, z_{1}, \cdots, z_{n-1}\right\}$ is a $C T$-system. Thus, we only have to prove that $\left\{1, z_{1}, \cdots, z_{n}\right\}$ is a 
$T$-system on $I$. Assume this is not so; then, there is a nontrivial linear combination $g$ of the functions $1, z_{1}, \cdots, z_{n}$, that vanishes on a set $t_{0}<t_{1}<\cdots<t_{n}$ of points of $I$. Since by inductive hypothesis $\left\{1, z_{1}, \cdots, z_{n-1}\right\}$ is a $T$-system on $I$, the coefficient of $z_{n}$ in $g$ cannot be zero; thus, without any loss of generality, we can assume it to equal 1. Since $B$ is dense in $I$, and $\left\{1, z_{1}, \cdots, z_{n}\right\}$ is a $T$-system thereon, there is a point $t_{n}^{\prime}$ of $B, t_{n-1}<t_{n}^{\prime}<t_{n}$, such that $g\left(t_{n}^{\prime}\right) \neq 0$. Assume first that $g\left(t_{n}^{\prime}\right)>0$; then, since the coefficient of $z_{n}$ in $g$ is 1 , developing by the last row and applying the inductive hypothesis, we see that

$$
\begin{aligned}
D\left(1, z_{1}, \cdots, z_{n} / t_{1}, \cdots, t_{n-1}, t_{n}^{\prime}, t_{n}\right) & =D\left(1, z_{1}, \cdots, z_{n-1}, g / t_{1}, \cdots, t_{n-1}, t_{n}^{\prime}, t_{n}\right) \\
& =-g\left(t_{n}^{\prime}\right) \cdot D\left(1, z_{1}, \cdots, z_{n-1} / t_{1}, \cdots, t_{n}\right) \\
& <0 .
\end{aligned}
$$

If $g\left(t_{n}^{\prime}\right)<0$, we similarly see that $D\left(1, z_{1}, \cdots, z_{n} / t_{0}, \cdots, t_{n-1}, t_{n}^{\prime}\right)<$ 0 . But these conclusions contradict the fact that $\left\{1, z_{1}, \cdots, z_{n}\right\}$ is a $W T$-system on $I$.

(c) The assertion is obviously true for $n=1$. Assume it to be true for $n=k$, and let $n=k+1$. Assume for example that $y_{1}$ is right continuous at a point $s$ of $A$. By inductive hypothesis, the functions $y_{t}$, $i=1, \cdots, n$ are right continuous at $s$. Let $t_{0}<t_{1}<\cdots<t_{n-1}<s<t_{n}$, where the $t_{i}$ 's are points of $A$. Since $D\left(1, y_{1}, \cdots, y_{n+1} / t_{0}, \cdots, t_{n-1}, s, t_{n}\right)$ is nonnegative, making $t_{n}$ tend to $s$, we see that

$$
\begin{aligned}
0 & \leqq D\left(1, y_{1}, \cdots, y_{n+1} / t_{0}, \cdots, t_{n-1}, s, s^{+}\right) \\
& =\left[y_{n+1}^{+}(s)-y_{n+1}(s)\right] \cdot D\left(1, y_{1}, \cdots, y_{n} / t_{0}, \cdots, t_{n-1}, s\right) .
\end{aligned}
$$

Thus $y_{n+1}^{+}(s)-y_{n+1}(s) \geqq 0$.

By considering now points $t_{i}^{\prime}$, such that $t_{0}^{\prime}<\cdots<t_{n-2}^{\prime}<s<t_{n-1}^{\prime}<t_{n}^{\prime}$ (if $n=2$, such that $s<t_{0}^{\prime}<t_{1}^{\prime}$ ), and making $t_{n-1}$ tend to $s$, we similarly see that $y_{n+1}^{+}(s)-y_{n+1}(s) \leqq 0$, whence the conclusion follows.

(d) The assertion is true by hypothesis for $n=2$. Assume it to be true for $n=k$, and let $n=k+1$. Let $s$ be any given point of $A$, and let $t_{0}<\cdots<t_{n}$ be points of the set $A \cap(s, b)$. Let $Q=$ $D\left(1, y_{1}, \cdots, y_{n} / t_{0}, \cdots, t_{n}\right)$ and, for $i=0,1, \cdots, n$, let the functions $z_{\imath}$ be defined by $z_{1}(t)=D\left(1, y_{1}, \cdots, y_{n} / t_{0}, \cdots, t_{1-1}, t, t_{1+1}, \cdots, t_{n}\right)$. It is readily seen that $D\left(z_{0}, \cdots, z_{n} / t_{0}, \cdots, t_{n}\right)=Q^{n+1}>0$, and proceeding as in [7, Lemma 2], we conclude that $\left\{z_{0}, \cdots, z_{n}\right\}$ is a Tchebycheff system on $A$. If $z_{n+1}(t)=D\left(z_{0}, \cdots, z_{n}, y_{n+1} / t_{0}, \cdots, t_{n}, t\right)$, it is also clear that $z_{n+1} \in$ $C\left(z_{0}, \cdots, z_{n}\right)$ thereon. Let $s_{0}<\cdots<s_{i}$ be points of $\left(a, t_{0}\right) \cap A$, $(i<n)$. Then

$$
0<D\left(z_{0}, \cdots, z_{n} / s_{0}, \cdots, s_{i}, t_{i+1}, \cdots, t_{n}\right)=Q^{i+1} D\left(z_{0}, \cdots, z_{i} / s_{0}, \cdots, s_{i}\right),
$$


and it follows that $\left\{z_{0}, \cdots, z_{n}\right\}$ is a $C T$-system on $\left(a, t_{0}\right) \cap A$. It is similarly seen that also $\left\{z_{0}, \cdots, z_{n-2},-z_{n}\right\}$ is a $C T$-system on $\left(a, t_{0}\right) \cap A$, and that $z_{n+1} \in C\left(z_{0}, \cdots, z_{n-2},-z_{n}\right)$ thereon. Let $v_{\iota}=z_{i} / z_{0}$, and assume first that $v_{1}$ is continuous at $s$. From (c) we know that all the functions $v_{i}$ must be continuous at $s$. It is therefore clear that the functions $v_{\imath}$ admit of a representation of the form (6) at the point $s$, where $p$ can be any number in the interval $[0,1]$. On the other hand, were $v_{1}$ discontinuous at $s$, if a representation of the form (6) exists at all, $p$ must clearly be unique. Applying therefore the inductive hypothesis to the systems $\left\{1, v_{1}, \cdots, v_{n}\right\}$ and $\left\{1, v_{1}, \cdots, v_{n-2},-v_{n}, v_{n+1}\right\}$ we conclude that the functions $v_{1}, \cdots, v_{n+1}$ admit of a representation of the form (6) at the point $s$. In particular, since $1 / z_{0}$ is in the linear span of these functions, it also admits of this representation, and we readily conclude that $p\left[z_{0}(s) / z_{0}^{+}(s)\right]+$ $(1-p)\left[z_{0}(s) / z_{0}^{-}(s)\right]=1$. Setting $q=p\left[z_{0}(s) / z_{0}^{+}(s)\right]$, and bearing in mind that $z_{i}=z_{0} \cdot v_{i}$, the conclusion follows.

Proof of Theorem 1. We shall first consider the case in which $\left\{1, u_{1}, \cdots, u_{n}\right\}$ is a $C T$-system of bounded functions defined on a bounded open interval $(a, b)$, such that $u_{i}=(1 / 2)\left(u_{i}^{+}+u_{i}^{-}\right)$on $(a, b)$, for $i=1, \cdots, n+1$.

Given a real-valued function $u$ defined on $(a, b)$, let $u(k, \cdot)$ be given by

$$
\begin{aligned}
u(k, t) & =\int_{a}^{b} u(s) G_{k}(t-s) d s, \quad \text { where } \\
G_{k}(s) & =(k / \sqrt{2 \pi}) \exp \left[-(1 / 2) k^{2} s^{2}\right]
\end{aligned}
$$

Under the conditions imposed on the functions $u_{i}$, it can be shown that $\lim _{k \rightarrow \infty} u_{i}(k, \cdot)=u_{i}$ on $(a, b)$, for $i=0,1, \cdots, n$. To see this, extend the functions $u_{v}$ to the whole real line by stipulating that they should vanish outside of $(a, b)$. Setting $\sigma_{i}(t)=\int_{a}^{t} u_{i}(s) d s$, and taking into consideration that $G_{k}(t-s)=G_{k}(s-t)$, we see that

$$
u_{i}(k, t)=\int_{-\infty}^{\infty} G_{k}(s-t) d \sigma_{i}(s)=\int_{-\infty}^{\infty} G_{k}(s) d \sigma_{t}(s+t),
$$

and the conclusion follows from [5, Theorem 4].

From the Basic Composition Formula [3, pp. 14, 15], we know that for any fixed integer $k, k=1,2, \cdots$, the system $\left\{u_{0}(k, \cdot), \cdots, u_{n}(k, \cdot)\right\}$ is an $E C T$-system on $[a, b]$. Let $c$ be any given point of $(a, b)$. From [3, Ch. XI, Thm. 1.1] we conclude that by adding to each function $u_{i}(k, \cdot)$ a suitable linear combination of its predecessors, we obtain a system 


$$
y_{\imath}(k, \cdot)=u_{\imath}(k, \cdot)+\sum_{j=0}^{i-1} a_{i, j}(k) u_{j}(k, \cdot)
$$

satisfying the constraints $y_{i}^{(r)}(k, c)=0 ; r=0,1, \cdots, i-1 ; i=1,2, \cdots, n$.

Proceeding exactly as in the proof of [3, Ch. XI, Thm. 1.2], we see that the functions $y_{i}(k, \cdot)$ admit of a representation of the form $y_{0}(k, \cdot)=$ $w_{0}(k, \cdot) ; y_{1}(k, t)=w_{0}(k, t) \int_{c}^{t} w_{1}(k, s) d s$, and in general

$y_{l}(k, t)=w_{0}(k, t) \int_{c}^{t} w_{1}\left(k, s_{1}\right) \int_{c}^{s_{1}} w_{2}\left(k, s_{2}\right) \int_{c}^{s_{2}} \cdots \int_{c}^{s_{t-1}} w_{t}\left(k, s_{l}\right) d s_{\imath} d s_{i-1} \cdots d s_{1}$ on $[a, b]$, for $i=1,2, \cdots, n$, where the functions $w_{i}(k, \cdot)$ are strictly positive. Thus the functions $p_{i}(k, t)=\int_{c}^{t} w_{i}(k, s) d s$ are strictly increasing. Clearly,

(7) $y_{i}(k, t)=y_{0}(k, t) \int_{c}^{t} \int_{c}^{s_{1}} \cdots \int_{c}^{s_{t-1}} d p_{t}\left(k, s_{i}\right) d p_{i-1}\left(k, s_{i-1}\right) \cdots d p_{1}\left(k, s_{1}\right)$;

$$
i=1,2, \cdots, n \text {. }
$$

Let $a<t_{0}<t_{1}<\cdots t_{n-1}<c$, where the points $t_{t}$ are arbitrary but fixed. Let

$$
\begin{aligned}
& z_{l}(k, t)=D\left(u_{0}(k, \cdot), \cdots, u_{i}(k, \cdot) / t_{0}, \cdots, t_{i-1}, t\right) / \\
& D\left(u_{0}(k, \cdot), \cdots, u_{i-1}(k, \cdot) / t_{0}, t_{1}, \cdots, t_{i-1}\right) .
\end{aligned}
$$

If $\|f\|_{A}$ denotes the supremum of the function $|f|$, taken over the set $A$, it is obvious that the sequences $\left\{\left\|u_{i}(k, \cdot)\right\|_{[a, b]}\right\} ; k=1,2, \cdots$ are bounded. Thus, also the sequences $\left\{\left\|z_{i}(k, \cdot)\right\|_{[a, b]}\right\} ; k=1,2, \cdots$ are bounded. Moreover, from [7, Lemma 3] we conclude that the functions $z_{i}(k, \cdot) ; i=1,2, \cdots, n$ admit of a representation of the form

$$
\begin{aligned}
z_{l}(k, t)=w_{0}(k, t) \int_{t_{i-1}}^{t} w_{1}\left(k, s_{1}\right) \int_{b_{1}(k, i)}^{s_{1}} w_{2}\left(k, s_{2}\right) \cdots & \\
& \int_{b_{i-1}(k, i)}^{s_{i-1}} w_{\imath}\left(k, s_{i}\right) d s_{i} \cdots d s_{1} ;
\end{aligned}
$$

where $t_{0}<b_{t-1}(k, i)<b_{t-2}(k, i)<\cdots<b_{1}(k, i)<t_{i-1}$. Therefore, by comparing (7) and (8) we conclude that if $t \geqq c, 0 \leqq y_{i}(k, t) \leqq z_{i}(k, t)$. This implies that the sequences $\left\{\left\|y_{i}(k, \cdot)\right\|_{[c, b]}\right\} ; k=1,2, \cdots$ are bounded, whence we can easily show that the coefficients $a_{t, j}(k)$ are uniformly 
bounded in $i, j$ and $k$. Thus, there exist numbers $a_{i, 1}, a_{t, 2}, \cdots, a_{i, i}$, with $a_{l, i}=1$, and a sequence $\left\{k_{j}\right\} ; j=1,2, \cdots$ such that, for $i=1,2, \cdots, n$,

$$
\lim _{j \rightarrow \infty} y_{\imath}\left(k_{j}, \cdot\right)=\sum_{j=0}^{t} a_{i, j} u_{j} \equiv y_{\imath}^{*}
$$

on $(a, b)$. Clearly $\left\{1, y_{1}^{*}, \cdots, y_{n}^{*}\right\}$ is a $C T$-system on $(a, b)$. It remains to show that there exist strictly increasing functions $p_{1}, \cdots, p_{n}$ such that for $y_{1}, \cdots, y_{n}$ as in equations (3), $y_{i}=y_{\imath}^{*}, i=1, \cdots, n$.

We shall now restrict ourselves to the consideration of any closed subinterval $[\alpha, \beta]$ of $(a, b)$. The proof for the whole of $(a, b)$ will follow from the uniqueness of the functions $p_{i}$.

We shall consider two cases, according as to whether the functions $p_{\imath}\left(k_{,}, \cdot\right)$ are bounded on $[\alpha, \beta]$ uniformly in $\left\{k_{l}\right\}$, or not. The first case will give us the desired representation, whereas the second will be shown to be impossible.

Since the functions $p_{i}(k, \cdot)$ are monotone, in the first case we can apply Helly's first theorem to conclude that there are increasing functions $p_{i}$ and a subsequence $\left\{k_{\jmath}^{\prime}\right\}$ of $k_{\jmath}$, such that $\lim _{\jmath \rightarrow \infty} p_{i}\left(k_{\jmath}^{\prime}, \cdot\right)=p_{i}$ on $[\alpha, \beta]$, for $i=1, \cdots, n$. Passing to the limit under the integral sign in (7), we conclude from (9) that the functions $y{ }_{1}^{*}$ have a representation of the form (3) on $[\alpha, \beta]$. (This passage to the limit, in which a Riemann-Stieltjes integral is obtained, can be easily justified by means of Theorems 15.2 and 15.6 of [ 2 , Chapter 2]). The functions $p_{i}$ must be strictly increasing, for assume that some function $p_{i}$ is not strictly increasing. Then $p_{i}$ must take a constant value on some subinterval of $[\alpha, \beta]$. By a simple inductive procedure, involving the number of integrations, we see that under this condition $y_{i}^{*}$ must be in the linear span of the functions $1, y_{1}^{*}, \cdots, y_{i-1}^{*}$ thereon. But this contradicts the fact that $\left\{1, y_{1}^{*}, \cdots, y_{n}^{*}\right\}$ is a $T$-system. Setting therefore $y_{i}=y_{1}^{*}, i=1, \cdots, n$, the conclusion follows.

Assume now that for some $i$ the sequence $\left\{p_{i}(k, \cdot)\right\}$ is not uniformly bounded on $[\alpha, \beta]$, and let $m$ be the smallest such $i$. Since the sequence $\left\{a_{1,0}(k)\right\} ; k=1,2, \cdots$ is bounded, we easily conclude from (9) that $m>1$. Since the functions $p_{m}(k, \cdot)$ are increasing, we know that one of the sequences $\left\{p_{m}(k, \alpha)\right\}$ or $\left\{p_{m}(k, \beta)\right\} ; k=1,2, \cdots$ is unbounded. Among the several possibilities, let us choose for illustration purposes the case in which $\left\{p_{m}(k, \alpha)\right\}$ is unbounded and $\alpha<c<\beta$. By definition, the functions $p_{i}(k, \cdot)$ vanish at $c$ and are negative to the left of $c$. Hence, there exists a sequence $\left\{k_{r}\right\}$ and strictly increasing functions $p_{i}$, such that $\lim _{r \rightarrow \infty} p_{t}\left(k_{r}, \cdot\right)=p_{\imath} \quad$ on $\quad[\alpha, \beta] ; \quad i=1, \cdots, m-1, \quad$ and, if $t \leqq \alpha$, $\lim _{r \rightarrow \infty} p_{m}\left(k_{r}, t\right)=-\infty$. Let $I_{n}\left(t_{1}, t_{2}\right)$ denote the subset of $R^{n}$ defined by $t_{1} \leqq s_{n} \leqq s_{n-1} \leqq \cdots \leqq s_{1} \leqq t_{2}$. Then, from (7) we see that, if $t<\alpha$, 


$$
\begin{aligned}
& \left|y_{m}(k, t) / y_{0}(k, t)\right| \\
& \quad=\int_{I_{m}(t, c)} d p_{m}\left(k, s_{m}\right) \cdots d p_{1}\left(k, s_{1}\right) \\
& \quad=\int_{I_{m-1}(t, c)}\left[-p_{m}\left(k, s_{m-1}\right)\right] d p_{m-1}\left(k, s_{m-1}\right) \cdots d p_{1}\left(k, s_{1}\right) \\
& \quad \geqq \int_{I_{m-1}(t / \alpha)}\left[-p_{m}\left(k, s_{m-1}\right)\right] d p_{m-1}\left(k, s_{m-1}\right) \cdots d p_{1}\left(k, s_{1}\right) \\
& \quad \geqq\left[-p_{m}(k, \alpha)\right] \int_{I_{m-1}(t / \alpha)} d p_{m-1}\left(k, s_{m-1}\right) \cdots d p_{1}\left(k, s_{1}\right) .
\end{aligned}
$$

Thus, $\left|y_{m}^{*}(t)\right|=\lim _{r \rightarrow \infty}\left|y_{m}\left(k_{r}, t\right) / y_{0}\left(k_{r}, t\right)\right|=\infty$, which is impossible.

Let $u$ be bounded, and convex with respect to $\left\{u_{0}, \cdots, u_{n}\right\}$. If $u$ is contained in their linear span, in order to obtain a representation of the form (5) it will suffice to take $p_{n+1}=1$. If $u$ is not contained in the span of the functions $u_{t}$, it is easily seen that $\left\{v_{0}(k, \cdot), \cdots, v_{n}(k, \cdot), u(k, \cdot)\right\}$ is an $E C T$-system, and we can proceed as above.

In order to establish the uniqueness of the functions $p_{t}$, assume that the functions $y_{i}$ admit of a representation of the form (3) with respect to two sets of functions $\left\{p_{i}\right\}$ and $\left\{q_{i}\right\}$, with $p_{\imath}(c)=q_{i}(c)=0 ; \quad i=$ $1, \cdots, n$. Then $\int_{c}^{t} d p_{i}=p_{i}(t)$ and $\int_{c}^{t} d q_{i}=q_{t}(t)$. Without loss of generality we can assume that $y_{0}(t)=1$. Then $y_{1}(t)=p_{1}(t)=q_{1}(t)$, and

$$
\begin{aligned}
y_{i}(t) & =\int_{c}^{t} \int_{c}^{s_{1}} \cdots \int_{c}^{s_{t-2}} p_{t}\left(s_{i-1}\right) d p_{i-1}\left(s_{t-1}\right) \cdots d p_{1}\left(s_{1}\right) \\
& =\int_{c}^{t} \int_{c}^{s_{1}} \cdots \int_{c}^{s_{t-2}} q_{t}\left(s_{t-1}\right) d q_{t-1}\left(s_{t-1}\right) \cdots d q_{1}\left(s_{1}\right) ; \quad i=2, \cdots, n,
\end{aligned}
$$

whence the assertion follows by repeated application of $[2, \mathrm{Ch}$. II, Theorem 16.2]. In similar fashion, it can be shown that $p_{n+1}$ is uniquely determined (a.e.), up to a constant, on the set of points at which it is strictly increasing.

We have thus shown that the functions $p_{t}$ are uniquely determined (a.e.) by the functions $y_{1}, \cdots, y_{i}$. In order to establish part (c) of the theorem, assume that the functions $y_{1}, \cdots, y_{k}$ admit of the representations (2) and (3), and the functions $z_{1}, \cdots, z_{k}$ of a representation of the form (2) with respect to coefficients $b_{i j}$, and of a representation of the form (3) with respect to functions $q_{1}, \cdots, q_{k}$, where $u_{0}=y_{0}=z_{0}$, and $p_{\imath}(c)=q_{i}(c)=0, i=1, \cdots, k$. We have to show that $a_{i j}=b_{i l}$, and $p_{\imath}=q_{i}$, for $j=0, \cdots, i-1$, and $i=1, \cdots, k$. Since the systems $\left\{y_{0}, \cdots, y_{k}\right\}$ and $\left\{z_{0}, \cdots, z_{k}\right\}$ have both been obtained from the system $\left\{u_{0}, \cdots, u_{k}\right\}$ by 
means of a triangular linear transformation, it is clear that there exist coefficients $c_{i j}$, such that, for $i=1, \cdots, k, y_{i}=z_{i}+\sum_{i=0}^{i-1} c_{i j} \cdot z_{j}$. Since the functions $y_{t}$ and $z_{t}$ admit of representations of the form (3), it is clear that they vanish at the point $c$, whence from the preceding formula we conclude that $0=c_{i 0} \cdot z_{0}$. Since $z_{0}=u_{0}$, it is strictly positive. We thus conclude that $c_{i 0}=0$, and in particular that $y_{1}=z_{1}$.

The proof of the assertion now easily follows by an inductive argument involving the number of integrations, and the fact that the function $p_{i}$ is uniquely determined by the functions $y_{1}, \cdots, y_{1}$.

Let us now consider the general case. Since $u_{0}$ is strictly positive, and $u_{0}, u_{1}$ is a $T$-system, it is clear that the function $u_{1} / u_{0}$ is strictly increasing. Let $Q$ be the image of $A$ under the function $\operatorname{arctg}\left(u_{1} / u_{0}\right)$. Since this function is strictly increasing and bounded, it is clear that $Q$ is a bounded set of real numbers, having property (D).

For every element $t$ of $Q$, let $\mathrm{I}(t)$ be the least upper bound of the set of points of $Q$ that precede $t$, and $\mathfrak{u}(t)$ the greatest lower bound of the set of points of $Q$ that follow $t$. Clearly there is an at most denumerable set of points of $Q$ for which $\mathfrak{l}-\mathfrak{u}>0$. For every element $t$ of $Q$, let $q(t)=\mathfrak{I}(t)+\sum_{s<t}[\mathfrak{u}(s)-\mathfrak{l}(s)] . \quad$ It is readily seen that $q$ is a strictly increasing function that transforms $Q$ into a dense subset of a bounded open interval. Let $h(t)=q\left[\arctan \left(u_{1} / u_{0}\right)(t)\right]$, and consider the functions defined on $h(A)$ by $z_{i}(t)=u_{i}\left[h^{-1}(t)\right] / u_{0}\left[h^{-1}(t)\right], i=0,1, \cdots, n+1$. Clearly $\left\{z_{0}, \cdots, z_{n}\right\}$ is a $C T$-system on $h(A)$, and $z_{n+1} \in C\left(z_{0}, \cdots, z_{n}\right)$ thereon. Since $z_{0}=1$, and $h(A)$ is a dense subset of an open bounded interval $(a, b)$, we know from Theorem 2(b) that, if $z_{i}^{*}=(1 / 2)\left[z_{i}^{+}+z_{i}^{-}\right]$, then $\left\{1, z_{1}^{*}, \cdots, z_{n}^{*}\right\}$ is a $C T$-system on $(a, b)$, and $z_{n+1}^{*} \in C\left(1, z_{1}^{*}, \cdots, z_{n}^{*}\right)$ thereon. By a procedure similar to the one employed in the proof of Theorem 2(a), we readily see that the functions $z_{i}^{*}$ are bounded in every interval of the form $\left(a^{\prime}, b^{\prime}\right)$, with $a<a^{\prime}<b^{\prime}<b$. From the uniqueness of the representations (2) and (3), we readily see that the assertions proved in the first part of this proof are also valid without the condition of boundedness. Thus, if $c$ is a point in the set $A$, and $d=h(c)$, we know there is a system $\left\{1, y_{1}^{*}, \cdots, y_{n+1}^{*}\right\}$ admitting of the representation $y_{i}^{*}=$ $z_{i}^{*}+\sum_{j=0}^{i-1} a_{i, \jmath} z_{j}^{*}, i=1, \cdots, n+1$, on $(a, b)$, as well as of a representation of the form (3) thereon (where $c$ is replaced by $d$ ), and the validity of statements (a), (b), (c), and (d) readily follows from the fact that $z_{\imath}=z_{i}^{*}$, except for an at most denumerable set of points. In order to prove (e) first note that, if $t$ is a point of $B$, it is easily seen, from the basic properties of Riemann-Stieltjes integrals, that the functions $y_{1}^{*}$ admit of a representation of the form (6) at the point $s=h(t)$, whence the proof of the necessity readily follows. Conversely, let $S$ denote the set of points of $(a, b)$ at which the functions $z_{i}$ admit of a representation of the form (6), with $p$ in the interval $[0,1]$. By virtue of their integral representation, we know that the functions $y_{i}^{*}$ can be written in the form 


$$
y_{i}^{*}(t)=\int_{d}^{t} x_{i}(s) d p_{1}(s),
$$

on $(a, b)$, for $i=1, \cdots, n+1$, where $x_{i}$ is a function of bounded variation in every closed subinterval of $(a, b)$. In particular, note that, for every point $t$ of $(a, b), y_{1}^{*}(t)=p_{1}(t)-0=z_{1}^{*}(t)+a_{1,0)}$. The function $p_{1}$ can be redefined at every point of discontinuity so as to assume any desired value between $p_{1}^{+}$and $p_{1}^{-}$, without changing the values of the functions $y_{1}^{*}$ at points other than these points of discontinuity, with one possible exception: If one of the functions $x_{t}$ is discontinuous and right (left) continuous at a point $t_{0}$ at which also the function $p_{1}$ is discontinuous, then $p_{1}$ cannot be redefined to equal $p_{1}^{+}\left(\right.$or $p_{1}^{-}$) at this point, without affecting the existence of the integral (10) for all points $t$ such that $|t-d|>\left|t_{0}-d\right|$, as was shown in the first counterexample of $\S 2$.

Let therefore $p_{1}^{*}$ be defined to equal $z_{1}+a_{1,0}$ on the set of points of $S$ for which $p$ is neither 0 nor 1 , and to equal $p_{1}$ at all other points of $(a, b)$; for $i=1, \cdots, n+1$, define $v_{i}$ by means of the formula $v_{t}(t)=$ $\int_{d}^{t} x_{i}(s) d p_{1}^{*}(s)$, and let $g_{i}=z_{i}+\sum_{\jmath=0}^{i-1} a_{i, j} z_{j}$, where the coefficients $a_{i, j}$ are the same ones that appear in the representation of the functions $y_{1}^{*}$ in terms of the functions $z_{i}^{*}$. In the light of the remarks made in the preceding paragraph, and bearing in mind that $v_{1}=g_{1}$ on $S$, we see that $v_{i}=g_{i}$ thereon, for $i=1, \cdots, n+1$. Thus, $y_{i}(t)=y_{0}(t) \cdot v_{\iota}[h(t)]$, on $S$, and the conclusion follows.

Proof of Theorem 3. The proof of part (a) is essentially contained in the preceding discussion, and will therefore be omitted. In order to prove part (b), note that the implication (i) $\Rightarrow$ (ii) is a direct consequence of [8], whereas (ii) $\Rightarrow$ (iii) is trivial. In order to prove that (iii) $\Rightarrow$ (ii), note that in view of the result of [8] we can assume, without any loss of generality, that $\left\{u_{0}, \cdots, u_{n}\right\}$ is a $C T$-system on $A$. Thus, it is clear that $\left\{u_{0}^{*}, \cdots, u_{k}^{*}\right\}$ is a $W T$-system on $(a, b)$, for $k=0, \cdots, n$. Assume now that $u_{0}^{*}\left(s_{0}\right)=0$, for some point $s_{0}$ of $(a, b)$. We claim that $u_{i}^{*}\left(s_{0}\right)=0$, $i=0, \cdots, n$, in contradiction of (iii). We proceed by induction on $i$. The assertion is true by hypothesis, for $i=0$. Assume it to be true for $i$ smaller or equal to $m$, and let $i=m+1$. Let $a<t_{0}<\cdots<t_{m-1}<$ $s_{0}<t_{m}<b$, where the points $t_{i}$ are contained in $A$. Clearly $D\left(u_{0}^{*}, \cdots, u_{m}^{*} / t_{0}, \cdots, t_{m}\right)>0$. Thus, since $u_{i}^{*}\left(s_{0}\right)=0, i=0, \cdots, m$, it is clear that

$$
\begin{aligned}
0 & \leqq D\left(u_{0}^{*}, \cdots, u_{m+1}^{*} / t_{0}, \cdots, t_{m-1}, s_{0}, t_{m}\right) \\
& =-u_{m+1}^{*}\left(s_{0}\right) D\left(u_{0}^{*}, \cdots, u_{m}^{*} / t_{0}, \cdots, t_{m}\right)
\end{aligned}
$$

whence it is clear that $u_{m+1}^{*}\left(s_{0}\right) \leqq 0$. Choosing now the points $t_{i}$ so that $a<t_{0}<\cdots<t_{m}<s_{0}$, we similarly conclude that $u_{m+1}^{*}\left(s_{0}\right) \geqq 0$. 
In order to prove that (iii) $\Rightarrow$ (i), assume also in this case that $\left\{u_{0}, \cdots, u_{n}\right\}$ is a $C T$-system. In view of the proof carried out in the preceding paragraph, it is clear that $u_{0}^{*}$ is strictly positive throughout $(a, b)$. By continuity we conclude that $\left\{u_{0}^{*}, \cdots, u_{k}^{*}\right\}$ is a $W T$-system on $(a, b)$, for $k=1, \cdots, n$, and the proof is carried out inductively, using (as was done in Theorem 1 to prove that the functions $p_{i}$ are strictly increasing) the representations (4) and (5), and the Lemma.

Acknowledgement. The author is greatly indebted to Dr. R. P. Kurshan of Bell Laboratories for his deep and useful comments concerning the organization of this paper, as well as for bringing to his attention a number of mistakes.

\section{REFERENCES}

1. M. W. Bartelt, Weak Chebyshev Sets and Splines, J. of Approximation Theory, 14 (1975), 30-37.

2. T. H. Hildebrandt, Introduction to the Theory of Integration; Academic Press, New York and London, 1963.

3. S. Karlin and W. Studden, Tchebycheff Systems, with Applications in Analysis and Statistics; Interscience, New York, 1966.

4. M. A. Rutman, Integral Representation of Functions Forming a Markov Series; Dokl. Akad. Nauk. SSSR 164 No. 5 (1965), 989-992 [English translation: Soviet Mathematics Doklady 6, Amer. Math. Soc. (1965), 1340-1343].

5. H. S. Shapiro, Smoothing and Approximation of Functions, Van Nostrand Reinhold, New York, 1969.

6. R. A. Zalik, Smoothness Properties of Generalized Convex Functions, Proc. Amer. Math. Soc., 56 (1976), 118-120.

7. — Existence of Tchebycheff extensions; J. Math. Anal. Appl., 51 (1975), 68-75.

8. R. Zielke, On Transforming a Tchebyshev-System into a Markov-System, J. of Approximation Theory, 9 (1973), 357-366.

9. — - Alternation Properties of Tchebyshev-Systems and the Existence of Adjoined Functions, J. of Approximation Theory, 10 (1974), 172-184.

Received January 7, 1975 and in revised form November 22, 1976.

Ben Gurion University of the Negev

BEERSHEVA 84 120, IsRaEL 


\section{PACIFIC JOURNAL OF MATHEMATICS}

\section{EDITORS}

RichaRd ARENS (Managing Editor)

University of California

Los Angeles, CA 90024

\section{R. A. Beaumont}

University of Washington

Seattle, WA 98105

C. C. Moore

University of California

Berkeley, CA 94720
J. DugunduI

Department of Mathematics

University of Southern California

Los Angeles, CA 90007

R. Finn AND J. Milgram

Stanford University

Stanford, CA 94305

\section{ASSOCIATE EDITORS}
E. F. BECKENBACH
B. H. NeumanN
F. WOLF
K. YoshidA

\section{SUPPORTING INSTITUTIONS}

UNIVERSITY OF BRITISH COLUMBIA

CALIFORNIA INSTITUTE OF TECHNOLOGY

UNIVERSITY OF CALIFORNIA

MONTANA STATE UNIVERSITY

UNIVERSITY OF NEVADA

NEW MEXICO STATE UNIVERSITY

OREGON STATE UNIVERSITY

UNIVERSITY OF OREGON

OSAKA UNIVERSITY

\author{
UNIVERSITY OF SOUTHERN CALIFORNIA \\ STANFORD UNIVERSITY \\ UNIVERSITY OF HAWAII \\ UNIVERSITY OF TOKYO \\ UNIVERSITY OF UTAH \\ WASHINGTON STATE UNIVERSITY \\ UNIVERSITY OF WASHINGTON \\ AMERICAN MATHEMATICAL SOCIETY
}

The Supporting Institutions listed above contribute to the cost of publication of this Journal, but they are not owners or publishers and have no responsibility for its contents or policies.

Mathematical papers intended for publication in the Pacific Journal of Mathematics should be in typed form or offset-reproduced (not dittoed), double spaced with large margins. Underline Greek letters in red, German in green, and script in blue. The first paragraph or two must be capable of being used separately as a synopsis of the entire paper. Items of the bibliography should not be cited there unless absolutely necessary, in which case they must be identified by author and Journal, rather than by item number. Manuscripts, in duplicate, may be sent to any one of the four editors. Please classify according to the scheme of Math. Reviews, Index to Vol. 39. All other communications should be addressed to the managing editor, or Elaine Barth, University of California, Los Angeles, California, 90024.

100 reprints are provided free for each article, only if page charges have been substantially paid. Additional copies may be obtained at cost in multiples of 50 .

The Pacific Journal of Mathematics is issued monthly as of January 1966. Regular subscription rate: $\$ 72.00$ a year (6 Vols., 12 issues). Special rate: $\$ 36.00$ a year to individual members of supporting institutions.

Subscriptions, orders for back numbers, and changes of address should be sent to Pacific Journal of Mathematics, 103 Highland Boulevard, Berkeley, California, 94708.

PUBLISHED BY PACIFIC JOURNAL OF MATHEMATICS, A NON-PROFIT CORPORATION

Printed at Jerusalem Academic Press, POB 2390, Jerusalem, Israel. 


\section{Pacific Journal of Mathematics}

Vol. 68, No. 2

April, 1977

William Allen Adkins, Aldo Andreotti and John Vincent Leahy, An analogue of Oka's theorem for weakly normal complex spaces ........

Ann K. Boyle, M. G. Deshpande and Edmund H. Feller, On nonsingularly

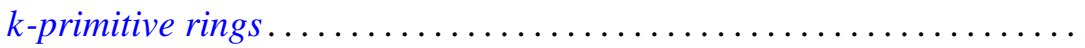

Rolando Basim Chuaqui, Measures invariant under a group of

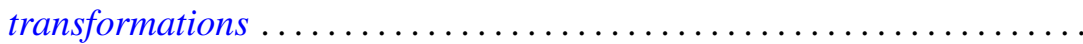

Wendell Dan Curtis and Forrest Miller, Gauge groups and classification of bundles with simple structural group .......................

Garret J. Etgen and Willie Taylor, The essential uniqueness of bounded nonoscillatory solutions of certain even order differential equations

Paul Ezust, On a representation theory for ideal systems

Richard Carl Gilbert, The deficiency index of a third order operator ........

John Norman Ginsburg, $S$-spaces in countably compact spaces using Ostaszewski's method.

Basil Gordon and S. P. Mohanty, On a theorem of Delaunay and some

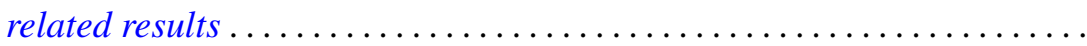

Douglas Lloyd Grant, Topological groups which satisfy an open mapping

theorem.

Charles Lemuel Hagopian, A characterization of solenoids

Kyong Taik Hahn, On completeness of the Bergman metric and its

subordinate metrics. II .

G. Hochschild and David Wheeler Wigner, Abstractly split group extensions.

Gary S. Itzkowitz, Inner invariant subspaces ...............

Jiang Luh and Mohan S. Putcha, A commutativity theorem for

non-associative algebras over a principal ideal domain.

Donald J. Newman and A. R. Reddy, Addendum to: "Rational approximation of $e^{-x}$ on the positive real axis".....

Akio Osada, On the distribution of a-points of a strongly annular function ....

Jeffrey Lynn Spielman, A characterization of the Gaussian distribution in a Hilbert space. .

Robert Moffatt Stephenson Jr., Symmetrizable-closed spaces ...

Peter George Trotter and Takayuki Tamura, Completely semisimple inverse $\Delta$-semigroups admitting principal series . . . . . . . .

Charles Irvin Vinsonhaler and William Jennings Wickless, Torsion free abelian groups quasi-projective over their endomorphism rings...

Frank Arvey Wattenberg, Topologies on the set of closed subsets ... 